\title{
ORIGINAL
}

\section{ESTADO NUTRICIONAL E INGESTÃO ALIMENTAR DE PESSOAS COM FIBROSE CÍSTICA}

\section{NUTRITIONAL STATUS AND FOOD INTAKE OF INDIVIDUALS WITH CYSTIC FIBROSIS}

\author{
Giovanna Medeiros Rataichesck FIATES' \\ Eliana BARBOSA ${ }^{2}$ \\ Flávia AULER ${ }^{3}$ \\ Simone Flach FEITEN ${ }^{4}$ \\ Fabiane MIRANDA ${ }^{4}$
}

\begin{abstract}
RESUMO
A mucoviscidose é uma doença que afeta o estado nutricional por interferir na ingestão e absorção adequadas de nutrientes. Este estudo avaliou 22 pacientes mucoviscidóticos atendidos no Ambulatório de Nutrição do Hospital Infantil Joana de Gusmão em Florianópolis, SC, no período de agosto de 1998 a janeiro de 1999. O estado nutricional foi determinado através de medidas antropométricas e dados de consumo alimentar. Quanto ao estado nutricional, 42,9\% dos menores de 2 anos e 28,6\% dos maiores de 10 anos estavam desnutridos; na faixa etária de 2 a 10 anos não houve nenhuma criança desnutrida. Quanto ao consumo alimentar, 33,3\% atingiram a recomendação de energia e 95,2\% atingiram a recomendação de proteína. Podemos concluir que a mucoviscidose afeta o estado nutricional das crianças em períodos críticos de crescimento e desenvolvimento, e que a qualidade da dieta destes pacientes pode ser melhorada através de um cuidado nutricional adequado.
\end{abstract}

Termos de indexação: nutrição, fibrose cística, avaliação nutricional, ingestão de alimentos, criança.

\begin{abstract}
Cystic fibrosis affects nutritional status because it interferes with adequate ingestion and absorption of nutrients. The present study evaluated 22 fibrocystic patients, who attended ambulatory visits at Hospital Infantil Joana de Gusmão, in the city of Florianópolis, Santa Catarina, Brazil, from August 1998 to January 1999. Nutritional status was determined by anthropometric measurements and food intake data. As to the nutritional status, $42.9 \%$ of the children under 2 years old and $28.6 \%$ over 10 years old were malnourished. Children between 2-10 years old did not present malnutrition. Evaluation of nutritional intake showed that $33.3 \%$ were eating adequate amounts of energy, and $95.2 \%$ of protein. It can be concluded that cystic fibrosis affects nutritional status of children in critical periods of growth and development, and that the quality of their diets can benefit from adequate nutritional care
\end{abstract}

Index terms: nutrition, cystic fibrosis, nutrition assessment, eating, child.

\footnotetext{
(1) Departamento de Nutrição, Centro de Ciências da Saúde, Universidade Federal de Santa Catarina. Campus Universitário, Trindade, 88040-900, Florianópolis, SC Brasil. Correspondência para/Correspondence to: G.M.R. FIATES.

(2) Hospital Infantil Joana de Gusmão, Florianópolis, SC.

(3) Mestranda do Curso de Pós-Graduação em Ciência dos Alimentos, Faculdade de Engenharia de Alimentos, Universidade Estadual de Campinas

(4) Bolsistas do Programa PET/CAPES, Curso de Graduação em Nutrição, Universidade Federal de Santa Catarina.
} 


\section{INTRODUÇÃO}

A fibrose cística ou mucoviscidose é a doença hereditária autossômica recessiva resultante da transmissão de cópias anormais do gene do regulador transmembrana da fibrose cística (RTFC), localizado no cromossomo 7 (Elborn et al., 1991). No organismo, a proteína RTFC se localiza nas superfícies apicais das células epiteliais e é um canal de cloro. A disfunção do canal leva a um distúrbio do transporte de cloro através dos epitélios (superfície luminal) e a um influxo compensatório de sódio para manter a eletroneutralidade com conseqüente influxo de água, o que leva a desidratação da superfície celular, com formação do muco espesso característico da doença (Campos et al., 1996).

Uma em cada vinte e cinco pessoas da população carrega o gene defeituoso, que é expresso quando uma criança herda o gene RTFC defeituoso de ambos os genitores. Este fato é compatível com uma freqüência observada de aproximadamente 1 em cada 2500 nascidos vivos (Collins, 1992). No Brasil, estudos realizados em cinco estados do Sul e Sudeste estimam uma incidência de 1 : 10000 mil recém-nascidos caucasóides (Campos et al., 1996).

A doença caracteriza-se por uma extensa disfunção das glândulas exócrinas, a qual resulta em um vasto conjunto de manifestações e complicações. Os produtos de secreção das glândula mucosas no pulmão e no trato gastrintestinal apresentam alterações físicas, tornando-se marcadamente espessas e viscosas. As complicações clínicas associadas com essas alterações incluem o desenvolvimento de bronquite crônica supurativa com destruição do parênquima pulmonar, insuficiência pancreática (levando a má-absorção e desnutrição), diabetes mellitus, doença hepática e comprometimento do sistema reprodutor masculino e feminino (Boat, 1988).

As complicações respiratórias são as principais causas de mortalidade e morbidade na fibrose cística (Penketh et al., 1987).

A má-absorção na fibrose cística é predominantemente ocasionada pela disfunção pré-epitelial e decorre da rejeição de nutrientes não hidrolisados no lúmen pela insuficiente secreção pancreática. Em 85\% dos pacientes firbrocísticos, o pâncreas não produz enzimas suficientes para completa digestão dos alimentos ingeridos, e as primeiras manifestações são a má digestão e má-absorção de gorduras. Os ductos pancreáticos ficam obstruídos por muco espesso, impedindo o suco pancreático de atingir o duodeno. A diminuição ou ausência de enzimas pancreáticas conduz à deficiência na absorção de lipídios, proteínas e, em menor escala, de carboidratos, causando esteatorréia, azotorréia e perda de vitaminas lipossolúveis. A baixa concentração de bicarbonato no suco pancreático faz com que o pH do duodeno seja ácido, e isso contribui para a má-absorção (Castro \& Dani, 1993).
O tecido endócrino do pâncreas é preservado inicialmente, mas com o aumento da idade do paciente células são perdidas e a glândula começa a ser completamente substituída por tecido fibroso e gordura (Creveling et al., 1997). Quando o comprometimento atinge a porção endócrina do pâncreas, pode ocorrer intolerância a glicose e diabetes mellitus. Isto ocorre em 8 a $15 \%$ dos pacientes, podendo aumentar com a idade (Donald, 1996). O diabetes, por sua vez, pode aumentar as perdas energéticas como um resultado da glicosúria (Ramsey et al., 1992).

A má nutrição na fibrose cística constitui um dos mais graves e difíceis desafios no manuseio dos enfermos. O quadro policarencial que vai se instalando é quase uma constante, mas não é uniforme e pode manifestar-se por facetas variadas, entre as quais: parada do crescimento, emagrecimento acentuado, deficiências nutricionais específicas, puberdade retardada, e grande comprometimento da função pulmonar (Zemel et al., 1996).

Em termos de alimentação, o consumo energético médio por dia no paciente chega a ser menor que $80 \%$ das recomendações internacionais da Recommended Dietary Allowances (RDA) (National Research..., 1989) e há evidências demonstrando que a manutenção de padrões nutricionais normais, não se faz enquanto a energia alimentar não atinja o mínimo de $130 \%$; para a retomada do crescimento, o mínimo energético deve atingir 150\% das recomendações da RDA de 1989. Macro e micronutrientes devem ser particularmente considerados na correção dos padrões nutricionais (Durie \& Pencharz, 1992; Damaceno \& Kierstman, 1995; Creveling et al., 1997).

Os problemas nutricionais e conseqüências da fibrose cística são multifatoriais e relacionadas com a progressão da doença. Fatores interdependentes, como deteriorização da função pulmonar, anorexia, vômitos, insuficiência pancreática e complicações biliares e intestinais, são responsáveis pelo aumento das necessidades energéticas, ingestão diminuída e aumento das perdas atribuídas a inadequação nutricional com conseqüente perda da massa magra e depressão da função imunológica (Creveling et al., 1997).

A intervenção nutricional na fibrose cística é de grande importância. Primeiramente, está associada com o melhor crescimento e melhora ou estabilização da função pulmonar. Em segundo lugar, a desnutrição tem muitos efeitos na função pulmonar, incluindo: diminuição na ventilação, na função muscular, na tolerância a exercícios e alterando a resposta imunológica pulmonar. Por fim, uma dieta rica em energia e gordura está associada a um melhor crescimento e maior sobrevida (Donald, 1996).

Nos últimos 30 anos, houve aumento marcante na expectativa de vida dos portadores de fibrose cística. Atualmente, a sobrevida de 80 a $90 \%$ dos pacientes chega a ser superior aos 20 anos de idade, aumentando à medida que precocemente se faz o diagnóstico (Green et al., 
1995). Assim, a fibrose cística não é mais uma doença exclusivamente pediátrica, e muitos pacientes estão sobrevivendo produtivamente na vida adulta (Creveling et al., 1997; Hudson \& Guill, 1998).

O objetivo deste trabalho foi o de caracterizar o estado nutricional e a ingestão alimentar de crianças e adolescentes com fibrose cística em tratamento ambulatorial por equipe multiprofissional.

\section{CASUÍSTICA E MÉTODOS}

A população estudada foi constituída de crianças, adolescentes e adultos com fibrose cística atendidos no Ambulatório de Nutrição do Hospital Infantil Joana de Gusmão, Florianópolis, Santa Catarina, Brasil, no período de agosto de 1998 a janeiro de 1999. Neste período foi iniciado o atendimento ambulatorial por nutricionista a pacientes com fibrose cística no referido hospital.

Todos os pacientes faziam parte da Associação Catarinense de Mucoviscidóticos (ACAM) no período do estudo. Esta associação é uma instituição que oferece apoio financeiro na compra de medicamentos e suplementos nutricionais a estes pacientes. Prestam serviços a esta associação uma equipe multidisciplinar, a qual conta com médicos (pneumologistas, cardiologistas e gastroenterologistas), nutricionista e fisioterapeuta, todos exercendo suas funções no Hospital Infantil Joana de Gusmão, Florianópolis, SC.

Durante a primeira consulta ambulatorial dos pacientes, foram coletados dados gerais (nome, data de nascimento, idade do diagnóstico, sexo, procedência); dados antropométricos (Peso ao Nascer (PN), Peso Atual $(\mathrm{Pa})$, Estatura $(\mathrm{E})$, Prega Cutânea Tricipital (PCT) e Circunferência do Braço $(\mathrm{CB})$ e dados referentes à ingestão alimentar através do recordatório de 24 horas.

Os dados acima foram sistematizados, para posterior análise, da seguinte forma:

\section{Dados de identificação}

a) Idade: corrigida de acordo com a quantidade de dias do mês corrente, isto é, um período igual ou inferior a quinze dias pertence ao mês anterior; e ao mês seguinte quando o período de dias transcorrido for superior a quinze dias do próximo mês (Moura, 1990; Vasconcelos, 1995);

b) Procedência: baseado na cidade a qual a família habita, os pacientes foram agrupados em três regiões: Grande Florianópolis, região Norte do Estado Vale do Itajaí e restante do Estado de Santa Catarina.

\section{Dados antropométricos}

a) Peso ao nascer: classificado de acordo com os critérios propostos por Nóbrega (1998), no qual fica estabelecido que recém-nascidos com mais de $3000 \mathrm{~g}$ possuem peso adequado (PA), entre 2500 e $2999 \mathrm{~g}$ peso insuficiente (PI), entre 1500 e 2499 g Baixo Peso ao Nascer (BPN) e com menos de 1500 gr Muito Baixo Peso ao Nascer (MBPN).

b) Indicadores antropométricos: crianças menores de 2 anos foram classificadas conforme o peso ideal para a idade (P/I), de acordo com o critério de Gomez (Gomez, 1956); as crianças de 2 a 10 anos foram classificadas utilizando os índices altura ideal para idade $(\mathrm{A} / \mathrm{I})$ e o peso ideal para altura $(\mathrm{P} / \mathrm{A})$ combinados, conforme o critério de Waterlow (1976). Para os pré adolescentes (10 a 12 anos), adolescentes (12 a 19 anos) e adultos, a avaliação foi feita através do Índice de Massa Corporal (IMC) por idade e sexo, conforme os critérios da Organização Mundial da Saúde (World Health..., 1995).

c) PCT e CB: foram avaliadas somente para maiores de 2 anos e comparadas com os critérios estabelecidos por Frisancho (1981).

\section{Ingestão alimentar}

a) Recordatório de 24 horas: entrevista feita com paciente e/ou auxílio da mãe no momento da consulta, com a quantificação de alimentos em medidas caseiras.

b) Cálculo dos totais ingeridos: realizado através do programa NUT (Programa..., 1995), no qual foi inserido o recordatório de 24 horas.

c) Adequação da ingestão alimentar (energia, proteínas, minerais e vitaminas): as necessidades energéticas e protéicas foram calculadas de acordo com Organización Mundial...(1985) e acrescidos 50\%; a adequação do consumo de minerais e vitaminas (cálcio, ferro, vitaminas A, E e K) comparada com as recomendações da RDA de 1989 acrescidas de 50\% (Durie \& Pencharz, 1992; Damaceno \& Kierstman, 1995; Creveling et al., 1997).

\section{RESULTADOS E DISCUSSÃO}

Foram coletados dados referentes a 22 pessoas com fibrose cística, englobando crianças de 5 meses a adultos de 20 anos e 5 meses perfazendo um total de $45,8 \%$ das pessoas atendidas pela Associação Catarinense de Mucoviscidóticos (ACAM) no período do estudo. O grupo foi constituído de 13 pessoas $(59,0 \%)$ do sexo feminino e 9 pessoas $(41,0 \%)$ do sexo masculino (Tabela 1).

Pode-se perceber que a maior parte do grupo $(68,2 \%)$ constituiu-se de crianças menores de 10 anos. Devido à manifestação precoce da doença e das complicações pulmonares, cardíacas e gastrintestinais, que elevam a morbidade, a mortalidade é grande antes que os pacientes atinjam a idade adulta (Farias et al., 1997). 
Tabela 1. Distribuição por idade e sexo, das pessoas com fibrose cística atendidas no Ambulatório de Nutrição do Hospital Infantil Joana de Gusmão, Florianópolis, SC, agosto de 1998 a janeiro 1999.

\begin{tabular}{|c|c|c|c|c|c|c|}
\hline \multirow{3}{*}{ Faixa etária } & \multirow{2}{*}{\multicolumn{2}{|c|}{ Distribuição por idade }} & \multicolumn{4}{|c|}{ Distribuição por sexo } \\
\hline & & & \multicolumn{2}{|c|}{ Masculino } & \multicolumn{2}{|c|}{ Feminino } \\
\hline & $\mathrm{n}$ & $\%$ & $\mathrm{n}$ & $\%$ & $\mathrm{n}$ & $\%$ \\
\hline$<2$ anos & 7 & 31,80 & 2 & 9,09 & 5 & 22,72 \\
\hline 2 anos a 5 anos & 3 & 13,70 & 1 & 4,54 & 2 & 9,09 \\
\hline 5 anos a 10 anos & 5 & 22,70 & 2 & 9,09 & 3 & 13,63 \\
\hline 10 anos a 15 anos & 3 & 13,70 & 2 & 9,09 & 1 & 4,54 \\
\hline$>15$ anos & 4 & 18,10 & 2 & 4,54 & 2 & 9,09 \\
\hline Total & 22 & 100,00 & 9 & 41,00 & 13 & 59,00 \\
\hline
\end{tabular}

Tabela 2. Estado nutricional das pessoas com fibrose cística atendidos no Ambulatório de Nutrição do Hospital Infantil Joana de Gusmão, Florianópolis, Santa Catarina, agosto de 1998 a janeiro de 1999.

\begin{tabular}{|c|c|c|c|c|}
\hline \multirow{2}{*}{ Classificação } & \multicolumn{2}{|c|}{ Sexo masculino } & \multicolumn{2}{|c|}{ Sexo feminino } \\
\hline & $\mathrm{n}$ & $\%$ & $\mathrm{n}$ & $\%$ \\
\hline Eutrofia & 7 & 31,8 & 10 & 45,4 \\
\hline Desnutrição/Magreza & 2 & 9,1 & 3 & 13,6 \\
\hline Total & 9 & 41,0 & 13 & 59,0 \\
\hline
\end{tabular}

Quanto à procedência dos pacientes, a maioria $(54,5 \%)$ foi oriunda da Região Norte do Estado de Santa Catarina, englobando o Vale do Itajaí, seguido da grande Florianópolis e do resto do Estado. A Região Norte e o Vale do Itajaí foram colonizadas por alemães e é justamente na população caucasiana a maior incidência desta doença. No Brasil, a ocorrência da fibrose cística é de aproximadamente 1 criança para cada 10000 nascidos vivos da raça branca (Damaceno \& Kierstman, 1995).

Em relação ao diagnóstico, 54,5\% fizeram-no antes de completar o primeiro ano de vida. Dados semelhantes foram encontrados no estudo realizado no Hospital das Clínicas da Universidade Federal do Paraná, onde $62,0 \%$ dos casos foram diagnosticados até 2 anos (Farias et al., 1997). O diagnóstico precoce é importante para a profilaxia das infecções pulmonares, para a reposição das enzimas pancreáticas, diminuição das complicações resultantes da doença e para o aumento da sobrevida (Campos et al., 1996).

Em relação ao estado nutricional, o grupo era composto, em sua maioria, por pacientes eutróficos (Tabela 2). Apenas 5 pacientes $(22,7 \%)$ foram classificados como desnutridos. No entanto, é interessante notar a faixa etária em que estes se encontravam: 3 deles menores de 2 anos, e 2 deles maiores de 10 anos.

Até completar 2 anos de idade, a criança passa por um período de rápido crescimento e conseqüente aumento das necessidades energéticas. Este fato, associado às complicações gastrintestinais e pulmonares, pode contribuir para a carência nutricional (Durie \& Pencharz, 1992; Ramsey et al., 1992).

Nenhum paciente na faixa etária de 2 a 10 anos apresentou estado nutricional comprometido, de acordo com os critérios de Waterlow (Waterlow, 1976). Nesta faixa etária as complicações pulmonares (pneumonias) e gastrintestinais (diarréias) são menos freqüentes, porque o diagnóstico já está concretizado e os pacientes já estão fazendo uso de enzimas e outras terapêuticas que auxiliam na melhora do estado nutricional (Damaceno \& Kierstman, 1995).

Quanto à situação nutricional dos pré-adolescentes, adolescentes e adultos, verifica-se que 28,6\% destes apresentaram magreza, provavelmente devido às inúmeras mudanças corporais, alteração de funções endócrinas, e acelerado crescimento aliado ao aumento do requerimento de nutrientes e atividade física intensa (Durie \& Pencharz, 1992; Ramsey et al., 1992).

Os dados da avaliação das medidas de PCT e CMB foram coletados apenas em pacientes maiores de 2 anos, perfazendo uma amostra de 13 pacientes. De acordo com os critérios usados (Frisancho, 1981), 30,7\% da população

Tabela 3. Estado nutricional, segundo critérios de Frisancho, para PCT e CMB das pessoas ( $>2$ anos) com fibrose cística atendidos no Ambulatório de Nutrição do Hospital Infantil Joana de Gusmão, Florianópolis, SC, agosto de 1998 a janeiro de 1999.

\begin{tabular}{lcc}
\hline Classificação & $\mathrm{n}$ & $\%$ \\
\hline $\mathrm{PCT}<$ 5o (depleção de tecido adiposo) & 4 & 30,7 \\
$\mathrm{PCT}>$ 5o (reservas normais de tecido adiposo) & 9 & 69,3 \\
\hline Total & 13 & 100,0 \\
\hline $\mathrm{CMB}<5^{\text {o }}$ (depleção muscular) & 6 & 46,1 \\
$\mathrm{CMB}>$ 50 (massa muscular normal) & 7 & 53,9 \\
\hline Total & 13 & 100,0 \\
\hline
\end{tabular}


estudada apresentou depleção significativa de tecido adiposo, ou seja, medida da PCT abaixo do percentil 5. A redução ou manutenção de peso e estatura compromete a conservação de tecido adiposo e muscular, acarretando a depleção (Mahan \& Arlin, 1995) (Tabela 3).

Em relação a presença de tecido muscular pode-se perceber que a depleção foi mais freqüente quando comparada com a reserva de tecido adiposo. Cerca de $46 \%$ da população apresentou déficit de tecido muscular, pois em caso de estado nutricional inadequado, o primeiro tecido a ser atingido é o muscular (Mahan \& Arlin, 1995).

Estudos de turnover protéico sugerem que pessoas com fibrose cistíca estão em um estado crônico de estresse catabólico ou desnutrição ou ambos, ligados a recorrentes exacerbações pulmonares que afetam adversamente o balanço energético e o metabolismo protéico. Estas mudanças podem afetar o curso da doença pulmonar por afetarem a função do músculo respiratório, exercício de tolerância e mecanismos de defesa pulmonar (Shepherd et al., 1986).

A PCT e a CMB usadas isoladamente não são indicadores precisos do estado nutricional, porém quando usados aliados a outros indicadores antropométricos ou como instrumentos de monitoração são bastante válidos (Creveling et al., 1997).

Existe uma íntima relação entre a alteração da composição corporal, desnutrição e função pulmonar em fibrose cística, e estimativas da composição corporal incluindo o uso de pregas cutâneas são essenciais para identificar precocemente, em relação a critérios antropométricos, pacientes em risco de desnutrição e declínio da função pulmonar (Thomson et al., 1995).

Verificou-se que a ingestão de $150,0 \%$ das recomendações da RDA de 1989 para energia foi atingida por apenas 33,3\% da amostra. A porcentagem da amostra que não ingere as necessidades energéticas foi grande $(66,7 \%)$, fazendo com que o estado nutricional possa ser comprometido de tal forma que os pacientes acabam por desenvolver um quadro de emagrecimento, podendo resultar em desnutrição e retardo do crescimento (Durie \& Pencharz, 1992; Creveling et al., 1997) (Tabela 4).

Alguns trabalhos têm demonstrado que um suporte nutricional agressivo em crianças fibrocísticas desnutridas intervém favoravelmente no processo de declínio da função pulmonar (Levy et al., 1985; Shepherd et al., 1986; Nir et al., 1996).

Em relação à ingestão protéica somente uma pessoa $(4,8 \%)$ não atingiu a recomendação de $150,0 \%$ da RDA (National Research..., 1989), provavelmente pelo uso de fórmulas alimentares especiais, ricas em proteína, como foi verificado através do recordatório de 24 horas. Observou-se que a maioria dos pacientes (86,3\%) faz uso diário de suplementos alimentares para aumentar a densidade energética e o teor protéico da dieta, tais como dietas sintéticas poliméricas líquidas e em pó, além de módulos de gordura, proteína e polímero de glicose (Tabela 4).

Em relação à ingestão alimentar de minerais (cálcio e ferro) e vitaminas (A, E e K), a maioria da amostra atingiu as necessidades nutricionais com o acréscimo de 50\% (National Research..., 1989).

O ferro é um oligoelemento que, quando tem sua ingestão reduzida, pode acarretar um quadro de anemia (Mahan \& Arlin, 1995). A anemia por deficiência de ferro com baixos níveis de ferritina sérica é freqüentemente observada em pacientes com doença pulmonar avançada. Porém, também pode ser observada em pacientes estáveis (Durie et al., 1992).

Nove pacientes $(42,85 \%)$ apresentaram ingestão insuficiente de cálcio $(<150 \%$ RDA). Este mineral está

Tabela 4. Adequação da ingestão alimentar de energia e proteína, segundo RDA de 1989, das pessoas com fibrose cística atendidas no Ambulatório de Nutrição do Hospital Infantil Joana de Gusmão, Florianópolis, SC, agosto de 1998 a janeiro de 1999.

\begin{tabular}{|c|c|c|c|c|}
\hline \multirow{2}{*}{ Adequação } & \multicolumn{2}{|c|}{ Energia } & \multicolumn{2}{|c|}{$\begin{array}{l}\text { Proteína } \\
\end{array}$} \\
\hline & $\mathrm{n}$ & $\%$ & $\mathrm{n}$ & $\%$ \\
\hline$<150 \%$ & 14 & 66,7 & 1 & 4,8 \\
\hline$>150 \%$ & 7 & 33,3 & 20 & 95,2 \\
\hline Total & 21 & 100,0 & 21 & 100,0 \\
\hline
\end{tabular}

Tabela 5. Adequação da ingestão alimentar de minerais e vitaminas, segundo RDA de 1989, das pessoas com fibrose cística atendidas no Ambulatório de Nutrição do Hospital Infantil Joana de Gusmão, Florianópolis, SC, agosto de 1998 a janeiro de 1999.

\begin{tabular}{|c|c|c|c|c|c|c|}
\hline \multirow{2}{*}{ Minerais e Vitaminas } & \multicolumn{2}{|c|}{ Adequação < 150 \% } & \multicolumn{2}{|c|}{ Adequação > 150 \% } & \multicolumn{2}{|c|}{ Total } \\
\hline & $\mathrm{n}$ & $\%$ & $\mathrm{n}$ & $\%$ & $\mathrm{n}$ & $\%$ \\
\hline Cálcio & 9 & 42,85 & 12 & 57,14 & 21 & 100,0 \\
\hline Ferro & 7 & 33,33 & 14 & 66,66 & 21 & 100,0 \\
\hline Vitamina A & 3 & 14,28 & 18 & 85,71 & 21 & 100,0 \\
\hline Vitamina $\mathrm{E}$ & 1 & 4,76 & 20 & 95,23 & 21 & 100,0 \\
\hline Vitamina $\mathrm{K}$ & 6 & 28,57 & 15 & 71,42 & 21 & 100,0 \\
\hline
\end{tabular}


diretamente relacionado com a formação dos ossos e dentes, além de participar no crescimento e ser co-fator/ regulador em várias reações bioquímicas (Dutra-de-Oliveira \& Marchini, 1998). A deficiência deste mineral, por períodos prolongados pode levar a um retardo no crescimento, palpitações cardíacas, hipertensão, raquitismo e fraqueza muscular. Poucos pacientes apresentaram consumo deficiente das vitaminas A, E e K. Isto é um resultado positivo, visto que cada uma destas vitaminas exerce funções específicas no organismo (Tabela 5).

\section{CONCLUSÃO}

Os pacientes avaliados neste estudo estavam tendo contato pela primeira vez com uma equipe multidisciplinar, e para muitos deles foi a primeira consulta com um nutricionista. Dentro da equipe, o papel do nutricionista é importante no que diz respeito a orientação dietética, avaliação do estado nutricional, determinação das necessidades energéticas e hábitos alimentares.

A fibrose cística possui uma estreita relação com a desnutrição, pois o estado nutricional está diretamente relacionado com o aumento da taxa de metabolismo basal em pacientes fibrocísticos. Como o comprometimento do estado nutricional de parte dos pacientes analisados ocorreu em períodos críticos de crescimento e desenvolvimento, concluímos que a qualidade da dieta destes pacientes pode ser melhorada através de um cuidado nutricional adequado.

Ao nutricionista cabe a orientação na realização de uma dieta adequada em energia, proteína e demais nutrientes, de forma a promover o crescimento e desenvolvimento normais para a idade, além de melhorar a função pulmonar, gastrintestinal e imune, possibilitando assim maior sobrevida. Como a dieta dos pacientes estudados estava adequada em proteína mas não em energia, o contato com o nutricionista deverá corrigir este problema.

Existem evidências atuais de que a maior sobrevida ocorre em pacientes tratados em Centros onde há atuação de equipe multiprofissional. No Hospital Infantil Joana de Gusmão, a equipe multidisciplinar está se formando com o apoio da Associação Catarinense de Mucoviscidóticos (ACAM), promovendo palestras educativas e atendimento periódico a crianças e adolescentes portadores de fibrose cística, buscando identificar e intervir precocemente nos problemas relacionados ao estado nutricional e função pulmonar. Com estas ações a equipe acredita estar contribuindo para melhoria da saúde, qualidade de vida e maior sobrevida destas crianças e adolescentes.

\section{REFERÊNCIAS BIBLIOGRÁFICAS}

CAMPOS, J.V.M., DAMACENO, N., CARVALHO, C.R.R., KOTZE, L.M. Fibrose cística. Arquivos de Gastroenterologia, São Paulo, v.33, p.1-48, 1996. Edição Especial.
CASTRO, L.P., DANI, R. Defeitos entéricos da absorção. In: DANI, R., CASTRO, L.P. Gastroenterologia clínica. 3.ed. Rio de Janeiro : Guanabara Koogan, 1993. p.733-757.

COLLINS, F.S. Cystic Fibrosis: molecular biology and therapeutic implications. Science, Washington DC, v.256, n.5058, p.774-779, 1992.

CREVELING, S., LIGHT, M., GARDNER, P., GREENE, L. Cystic fibrosis, nutrition and the health care team. Journal of the American Dietetic Association, Chicago, v.97, n.10, p.186-191, 1997.

DONALD, A.M. Nutritional management of cystic fibrosis. Archives of Disease in Childhood, London, v.74, n.1, p.81-87, 1996.

DURIE, P.R., PENCHARZ, P.B. Nutrition deficiencies in cystic fibrosis. British Medical Bulletin, London, v.48, n.4, p.823-847, 1992.

DUTRA-DE-OLIVEIRA, J.E., MARCHINI, J.S. Ciências nutricionais. São Paulo : Sarvier, 1998. 403p.

ELBORN, J.S., SHALE, D.J., BRITTON, J.R. Cystic fibrosis: current survival and population estimates to the year 2000. Thorax, Denmark,v.46, n.12, p.881-885, 1991.

FARIAS, L., ROSÁRIO FILHO, N.A., KOVALHUK, L., MIASOK, N., CHAVES, S.M., RECCO, A.S., PADILHA, T.M.. Aspectos da fibrose cistica: experiência no hospital de clínicas da UFPR : 1980-1996. Pediatria, São Paulo, v.19, n.4, p.241-248, 1997.

FRISANCHO, A.R. New norms of upper limb fat and muscle areas for assessment of nutritional status. American Journal of Clinical Nutrition, Bethesda, v.34, n.11, p.2540-2545, 1981.

GOMEZ, F. Mortality in second and third degree malnutrition. Journal Tropical Pediatrics, Oxford, v.2, n.2, p.77-83, 1956.

GREEN, M.R., BUCHANAN, E., WEAVER, L.T. Nutricional management of the infant with cystic fibrosis. Archives of Disease in Childhood, London, v.72, n.5, p.452-456, 1995.

HUDSON, V.L., GUILL, M.F. New developments in cystic fibrosis. Pediatric Annals, New York, v.27, n.8, p.515-520, 1998.

LEVY, L.D., DURIE, P.R., PENCHARZ, P.B., COREY, M.L. Effects of long-term nutritional rehabilitation on body composition and clinical status in malnourished children and adolescents with cystic fibrosis. The Journal of Pediatrics, Toronto, v.107, n.2, p.225-230, 1985.

MAHAN, L.K., ARLIN, M.T. Krause: alimentos nutrição e dietoterapia. 8.ed. São Paulo : Roca, 1995. cap.33, p.620-624.

MOURA, E.F.A. Estado nutricional de crianças hospitalizadas. Jornal de Pediatria, Rio de Janeiro, v.66, n.10/12, p.243-246, 1990.

NATIONAL RESEARCH COUNCIL (USA). Recommended Dietary Allowances. 10.ed. Washington DC : National Academy Press, 1989. $284 \mathrm{p}$.

NIR, M., LANNG, S., JOHANSEN, H.K., KOCH, C. Long term survival and nutritional data in patients with cystic fibrosis treated in a Danish centre. Thorax, Denmark, v.51, n.10, p.1023-1027, 1996.

NOBREGA, F.J. Distúrbios da nutrição. Rio de Janeiro : Revinter, 1998. cap.17, p.109.

ORGANIZACIÓN MUNDIAL DE LA SALUD. Necessidad de energia y de proteinas. Genebra, 1985. 220p. (Informe de una Reunión Cosultiva Conjunta FAO/OMS/UNU). 
PENKETH, A.R.L., WISE, A., MEARNS, M.B. Cystic fibrosis in adolescents and adults. Thorax, Denmark, v.42, n.7, p.526-532, 1987.

RAMSEY, B.W., FARRELL, P.M., PENCHARZ, P. Nutritional assessment and management in cystic fibrosis: a consensus report. American Journal of Clinical Nutrition, Bethesda, v.55, n.1, p.108-116, 1992.

SHEPHERD, R.W., HOLT, T.L., THOMAS, B.J., KAY, L., ISLES, A., FRANCIS, J. WARD, C. Nutritional rehabilitation in cystic fibrosis: controlled studies of effects on nutritional growth retardation, body protein turnover and course of pulmonary disease. Journal of Pediatrics, St. Louis, v.109, n.5, p.788-794, 1986.

THOMSON, M.A., QUIRK, P., SWANSON, C.E., THOMAS, B., HOLT, T.L., FRANCIS, P.T., SHEPHERD, R.W. Nutritional growth retardation is associated with defective lung growth in cystic fibrosis: a preventable determinant of pulmonary disfunction. Nutrition, Burbank CA, v.11, n.4, p.350-354, 1995.
VASCONCELOS, F.A.G. Avaliação nutricional de coletividades. 2.ed. Florianópolis: UFSC, 1995. 146p.

WATERLOW, J.C. Classification and definition of protein-caloric malnutrition. In: BEATON, G.H., BENGOA, J.M. Nutrition in preventive medicine. Geneva : WHO, 1976. p.530-555 (Monograph, 62).

WORLD HEALTH ORGANIZATION. Expert committee on physical status: the use and interpretation of anthropometry. Geneva, 1995. 451p. (WHO Technical Report Series, 854).

ZEMEL, B.S., KAWCHAK, D.A., CNAAN, A., ZHAO, H., SCANLIN, T.F., STALLINGS, V.A. Prospective evaluation of resting energy expenditure, nutritional status, pulmonary function, and genotype in children with cystic fibrosis. Pediatric Research, Baltimore, v.40, n.4, p.578-86, 1996.

Recebido para publicação em 13 de agosto de 1999 e aceito em 31 de julho de 2000. 